\title{
Blood Transfusion Reaction in a Post-Operative Total Knee Replacement Patient Transfused with Red Blood Cells
}

\author{
Khanna $\mathrm{V}^{*}$ and Wani Z \\ ${ }^{1}$ Department of Orthopaedics, National Institute of Medical Sciences, Jaipur \\ 2Department of Anaesthesia and Critical care, National Institute of Medical Sciences, \\ Jaipur
}

*Corresponding author: Vikram Khanna, Department of Orthopaedics, National Institute of Medical Sciences, Shobha Nagar, Jaipur - 303121, India, Tel: +91 7073647974; E-mail: 86.khanna@gmail.com

\section{Abstract}

The discovery of the ABO blood group, over 100 years ago, caused great excitement. Until then, all blood had been assumed to be the same, and the often tragic consequences of blood transfusions were not understood. As our understanding of the ABO group grew, not only did the world of blood transfusion become a great deal safer, but scientists could now study one of the first human characteristics proven to be inherited. We hereby, report a case of postoperative total knee replacement surgery were patient had received different blood transfusion as we fail to identify the exact group. WHICH STILL REMAINS A MYSTERY?

Keywords: ABO blood group; Blood transfusion; Total knee replacement

\section{Introduction}

Transfusion medicine has become important in the life support of critically ill patients. Transfusion medicine is based upon various blood types. These are genetic markers on RBC surfaces which are specific to particular species and are highly antigenic. Hence, it may be said that specific antigens on a red blood cell surface determine the blood type. Blood types with two or more alleles comprise the blood group system in humans. Blood type is generally inherited from the parents and is determined by two components: the $\mathrm{ABO}$ system and $\mathrm{Rh}$ factor. The $\mathrm{ABO}$ system is divided into four blood types: A, B, AB and O. The blood type is based on the presence or absence of antigens in the blood. Proteins that stick to the surface of RBCs are the antigens. The type of antigen determines the blood type. Along with the ABO system, the blood type is also divided on the presence or the absence of another antigen which is known as the Rh factor.

\section{Donations work as follows}

- $\quad$ 0: These individuals can donate blood to any group (because of the absence of any ABO antigen in their blood), but they can receive blood only from other type 0 individuals.

- A: These individuals can donate blood to type A and also type $A B$ individuals. They individuals receive blood only from type $\mathrm{A}$ and type $\mathrm{O}$ individuals.

- B: These individuals can donate blood to type B and also type $A B$ individuals. They can receive blood only from type $B$ and type $O$ individuals.

- $\quad$ AB: These individuals can donate blood only to type $\mathrm{AB}$ individuals, but can receive blood of from any type.

It is of utmost importance to know the blood type to prevent any blood mismatch causing fatality during the transfusion. On mixing 2 different types of blood clumping of blood cells may occur as a transfusion reaction which can be potentially fatal. Hence, before giving a blood 


\section{Anaesthesia \& Critical Care Medicine Journal}

transfusion, testing of the blood type along with crossmatching against the donor blood, minimizes the risk of transfusion reaction.

\section{Transfusion reactions}

Generally, the practice of typing and cross matching prevents adverse transfusion reactions. However, "the wrong blood" due to some clerical error can result in being transfused to a patient can result in death of the patient $(1,2)$. If a patient with blood group 0 is transfused with non $\mathrm{O}$ group RBCs, the anti-A and anti-B antibodies in the recipient's blood bind to their corresponding antigens which are present on the transfused RBCs. This causes complement fixation causing rapid intravascular haemolysis which triggers an acute haemolytic transfusion reaction that may cause DIC, shock, acute renal failure, and death. Hence, in a blood transfusion it is essential for the individual to receive a blood type which is compatible to that of his/her own otherwise it may end disastrously. The antigens of the donor should match those of the recipient as the host immune system attacks any donor RBCs containing antigens different from its own. To check for a match small samples of the donor and the recipient blood are mixed together in a process called "cross-matching." In emergency, O-ve blood may be given if the situation is life-threatening or if the similar blood type is in short supply.

\section{Case Report}

A 64yrs male underwent total knee replacement surgery followed by which post- operatively he was given 2 Blood transfusion of Blood Group A+ve after cross matching, which was diagnosed pre- operatively in Routine examination. After about 30mins of transfusion, our patient started having shivering and shortness of breath and his vitals were:

- Blood pressure: 86/40 mmhg

- Oxygen saturation: $95 \%$

- Temperature: $37.9^{\circ} \mathrm{C}$

- Pulse: $120 / \mathrm{min}$.

Following by which the transfusion of $2^{\text {nd }}$ bag in between. Antihistaminics along with paracetamol and steroids were given intravenously and patient was oxygenated. After about 30-40 mins patient found relief and was again in his good health. After a day again Blood Transfusion was advised as post - operative haemoglobin was $8 \mathrm{gm} \%$. Before transfusion we cross matched blood and the first unit was started. Vital signs were recorded:

- Blood pressure: 130/72
- Oxygen saturation: $96 \%$

- Temperature: $36.9^{\circ} \mathrm{C}$

- Pulse: $109 / \mathrm{min}$.

This time too after sometime vitals which were noted was as followed: Blood pressure: $82 / 44 \mathrm{~mm} \mathrm{hg;} \mathrm{Oxygen}$ saturation: $90 \%$; Temperature: $38.4^{\circ} \mathrm{C}$; Transfusion was stopped. Blood sample of patient was sent again for grouping and this time it came out to be $B+v e$. To this confusion of $A+v e \& B+v e$ again blood sample was sent to our laboratory and this time it came out with the result of $A B+v e$. We could not confirm if it was $A+v e$ or $A B+v e$ because of various $A+v e$ blood transfusions in past. And we were also not in position to wait further as patient's hemoglobin had reduced to $6.4 \mathrm{gm} \%$ so it was decided to not take chance and $0+v e$ blood group was arranged and transfused in emergency. 0+ve was transfused as one thing what we were sure of was that $\mathrm{RH}$ factor is Positive as in all the types of blood groups diagnosed one thing which was common was Positive $\mathrm{RH}$ factor and the patient survived. But till now we are still unsure of the blood type that patient shared as none of the test gave us a single type. Each time our results varied.

\section{Discussion}

Blood transfusion should not be done just like that. With the advent of different components of blood, the risk and benefits of the blood components should be assessed and only the required components should be transfused. Transfusion reactions should be recognized immediately and aggressive and management may be required to save the patients' life. On the suspicion of a transfusion reaction, the transfusion should be stopped and the IV line is maintained with a normal saline drip. This is followed by checking the blood label and identifying whether the "right" blood unit was administered to the "right" patient. Acute transfusion reactions may present as a confounding presentation where it becomes difficult to differentiate between the reactions due to transfused blood or any complication of the illness due to which the blood has been transfused. Some serious infections may also be transmitted via the blood transfusions and hence, it is not only morally but legally required to inform the patients about all the risks which may be entailed in the transfusion of blood. The important thing to remember is that whenever the patient is undergoing blood transfusion and the patient has the following sign like:

- Shivering

- Fever

- Hypotension

- Rashes 


\section{Anaesthesia \& Critical Care Medicine Journal}

- Itching

- Difficulty in breathing

It is essential that a transfusion reaction should be suspected and the transfusion should be stopped immediately followed by parenteral anti histaminic and steroids should be injected. This is followed the re-cross matching of the blood and the patient is managed with plenty of parenteral fluid therapy with close monitoring on the vitals to detect immediately if there is any dip in the vital parameters.

\section{References}

1. Sazama K (1990) Reports of 355 transfusionassociated deaths: 1976 through 1985. Transfusion 30(7): 583-590.

2. Williamson LM, Lowe S, Love EM, Cohen H, Soldan K, et al. (1999) Serious hazards of transfusion (SHOT) initiative: analysis of the first two annual reports 319(7201): 16-19.

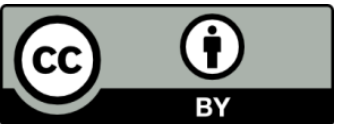

